\title{
Performance Enhancement of Noisy Distributed Acoustic Sensor Using Normalized Differential Method
}

\author{
Islam Ashry, ${ }^{\mathrm{a}, \dagger}$ Yuan Mao,,${ }^{\mathrm{a}, \dagger}$ Mohd Sharizal Alias, ${ }^{\mathrm{a}}$ Tien Khee Ng, ${ }^{\mathrm{a}}$ Frode Hveding, ${ }^{\mathrm{b}, \#}$ Muhammad \\ Arsalan, ${ }^{\mathrm{b}}$ and Boon S. Ooi ${ }^{\mathrm{a}, *}$ \\ ${ }^{a}$ King Abdullah University of Science and Technology (KAUST), Computer, Electrical, and \\ Mathematical Sciences and Engineering, Thuwal 23955-6900, Saudi Arabia \\ ${ }^{b}$ EXPEC Advanced Research Center, Saudi Aramco, Dhahran 34464, Saudi Arabia \\ ${ }^{\dagger}$ These authors contributed equally to this work \\ "\#frode.hveding@aramco.com, *boon.ooi@kaust.edu.sa
}

\begin{abstract}
We experimentally report a normalized differential signal processing technique to improve the signal-to-noise ratio (SNR) of a fiber optic distributed acoustic sensor (DAS), in the time-domain. The introduced method is calibrated through comparing it with the typical differential method when using a noisy DAS system that includes a relatively wide linewidth laser. For this system, the normalized differential method allows measuring the vibration locations, produced by a piezoelectric transducer (PZT) cylinder, with enhanced SNR.
\end{abstract}

Keywords: Acoustic sensors, optical fiber sensors, optical fibers, optical sensors.

\section{INTRODUCTION}

Optical fiber sensors are particularly attractive since they have numerous advantages including harsh environment operation, immunity to electromagnetic interference, and multiplexing [1]. One important example is the distributed acoustic sensor (DAS) designed using the phase-sensitive optical time domain reflectometry ( $\Phi$-OTDR) [2]. The $\Phi$ OTDR based DAS has been used in a myriad of applications, including real-time structural health monitoring [3], oil and gas industry [4], and aerospace transportation [5]. Briefly, its underlying operation principle relies on using a coherent (narrow linewidth) laser source to launch optical pulses into a fiber. While a pulse is propagating along the fiber, a Rayleigh trace is backscattered to be recorded at the fiber input port. By monitoring the intensity temporal evolution of the recorded Rayleigh traces, one can figure out a position along the fiber where subject to vibration and also the vibration amplitude and frequency [2].

In the conventional detection setups employed for the $\Phi$-OTDR based DAS, there are numerous noise sources including, laser phase noise and frequency drift, temporal pulse intensity fluctuation, photodetector thermal and shot noises, and low extinction ratio of the launched pulses [6]. These noise sources make the recorded Rayleigh traces unstable in the time-domain which consequently degrades the signal-to-noise ratio (SNR) of the DAS system. Averaging the Rayleigh traces is the typical signal processing method utilized to reduce the temporal intensity instability of the Rayleigh traces. However, the averaging method decreases the maximum vibration frequency to be detected. For instance, in case an averaged trace is determined using $M$ consecutive Rayleigh traces, the effective repetition frequency decreases to become the real repetition rate of the pump pulses divided by $M$. Consequently, following the sampling theory, the maximum vibration frequency to be detected (Nyquist frequency) is reduced down by a factor of $M$, compared with to the no averaging case. Instead, the moving averaging and moving differential algorithm can get around twice frequency response over that of the typical averaging method, but this is still significantly less than the Nyquist frequency [7].

In this paper, we report a simple signal processing method to enhance the time-domain SNR of the optical fiber DAS system. In this technique, without averaging, we use a normalized differential between the successive Rayleigh traces [8]. The effectiveness of our method is experimentally investigated using the typical direct detection scheme. The capabilities of the normalized differential method is determined via comparing it with the standard differential technique, when using noisy optical devices, such as a relatively wide linewidth laser. Using the introduced signal processing method, we can detect vibrations produced by a piezoelectric transducer (PZT) cylinder with improved SNR in the noisy DAS system. 


\section{DEFINITION OF THE NORMALIZED DIFFERENTIAL METHOD}

We here introduce the normalized differential method and further demonstrate its advantages over the conventional differential one typically used in the literature [7]. The used experimental setup of the optical fiber DAS is schematically presented in Fig. 1. We use a laser of a $100 \mathrm{kHz}$ linewidth and $1535 \mathrm{~nm}$ operation wavelength to generate a continuouswave $(\mathrm{CW})$ light of $\sim 10 \mathrm{~mW}$ optical power. This laser is considered a relatively noisy source for the optical fiber DAS because its linewidth is wide compared with the other light sources used in similar systems [2]. An electro-optic modulator (EOM1) is then used to modulate the $\mathrm{CW}$ light into optical pulses of $20 \mathrm{kHz}$ repetition rate and $100 \mathrm{~ns}$ width, which provides $10 \mathrm{~m}$ spatial resolution. Because the EOM1 is polarization-dependent, a polarization controller (PC1) is used to maximize the peak power of the optical pulses produced by the EOM1. In order to improve the extinction ratio of the optical pulses, we use another electro-optic modulator (EOM2) which is well-synchronized with the EOM1 via a function generator [9]. Similarly, another polarization controller (PC2) is used to enhance the peak power of the pulses generated by the EOM2. Afterwards, an erbium-doped-fiber-amplifier (EDFA1) amplifies the optical pulses which are then injected via a circulator into a standard single-mode fiber (SMF) of $4 \mathrm{~km}$ length. Near the SMF end, we attach a 10 $\mathrm{m}$ section of the fiber to a PZT cylinder such that its vibration amplitude and frequency can be controlled via a driven function generator. The backscattered Rayleigh signal from the fiber is amplified by another erbium-doped-fiberamplifier (EDFA2) which amplified spontaneous emission (ASE) noise is discarded using a fiber Bragg grating (FBG). Eventually, the backscattered Rayleigh signal is recorded via a photodetector (PD) and sampled using an oscilloscope.

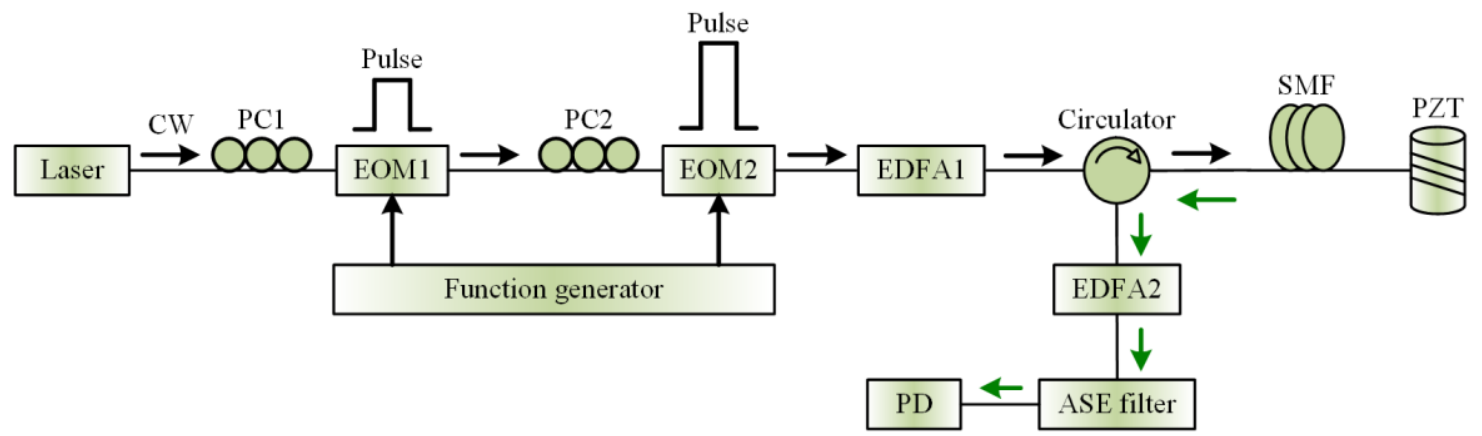

Figure 1. Schematic of the optical fiber DAS with direct detection.

We initially run our experiment while the PZT cylinder is static. In Fig. 2(a), we show a representative example of 100 successive Rayleigh traces. As presented in Fig. 2(a), for the individual traces, the backscattered Rayleigh signals from the fiber have various intensity amplitudes. The high intensity signals located at the start of the traces occur because of the fiber front facet reflection, not the Rayleigh scattering. Typically, there are two goals for an $\Phi$-OTDR based DAS: locating a position along the fiber subjected to a vibration, and determining the frequency components of the vibration. In the literature, the first target is conventionally calculated using the typical differential method, i.e., subtracting the successive Rayleigh traces. As shown in Fig. 2(b), we show the typical differential signal of the 100 successive Rayleigh traces. The results shown in Fig. 2(b) are basically the background noise of the used experimental setup. Clearly, the noise levels are not equal along the SMF length. Instead, the noise relies on the original intensity within the Rayleigh traces. For example, focusing on the behavior of two arbitrary high-intensity speckles (marked as L1 and L2) in Fig. 2(a). By calculating their corresponding noise levels, as illustrated in Fig. 2(b), these two speckles generate higher noise values than those of the lower intensities. This is to be expected because if assuming the percentage of a trace fluctuation is almost fixed along the entire SMF, the differences between the high intensity speckles should be larger than that of the low intensity ones. Assuming the worst case scenario which happens when a vibration occurs at a location along the SMF which originally generates a low intensity Rayleigh signal within the traces, the corresponding differential signal of this vibration might submerge into the noise background to mislead the vibration position. Instead, in this work, we report the normalized differential method that offers signals independent of the speckles intensities within the Rayleigh traces. The normalized differential trace is defined as $\Delta R_{\text {norm }}=\left(R_{i+1}-R_{i}\right) / R_{i}, i \in[1, N-1]$, where $R_{i}$ denotes the $i$ th Rayleigh trace and $N$ is the total number of traces. In Fig. 2(c), we show $\Delta R_{\text {norm }}$ of the 100 Rayleigh traces presented in Fig. 2(a). Compared with the results of Fig. 2(b), our technique significantly enhances the noise equalization along the entire SMF which consequently enables locating vibration positions in case if their Rayleigh signals are originally weak. 


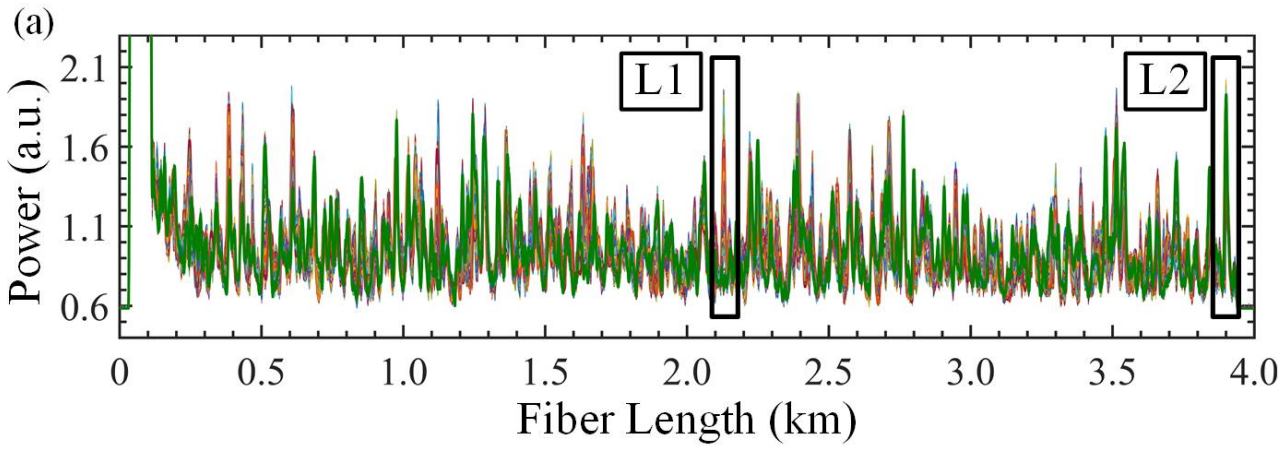

(b) Without normalization

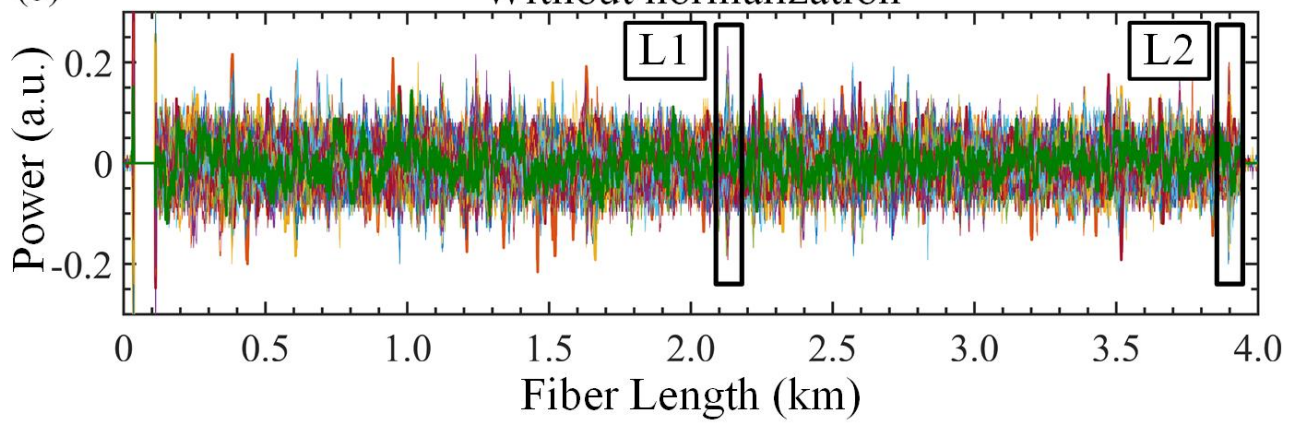

(c)

With normalization

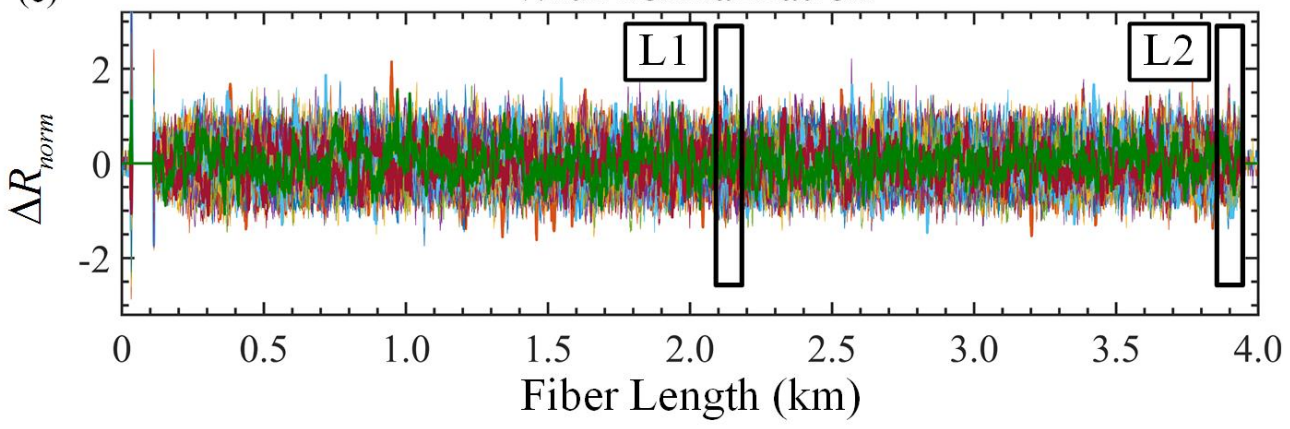

Figure 2. (a) Successive Rayleigh traces recorded when the PZT is static. Difference between the Rayleigh traces using the typical differential (b) and normalized differential (c) methods.

\section{SENSING OF PZT VIBRATIONS}

In order to explore the effectiveness of the introduced normalized differential method on the SNR of the $\Phi$-OTDR based DAS, we compare it with the conventional differential method as the PZT cylinder is vibrating (shown in Fig. 1). In Fig. 3(a), we show 100 successive Rayleigh traces recorded while the PZT cylinder is sinusoidally vibrating with $2 \mathrm{kHz}$ frequency. It is worth mentioning that the traces shown in Fig. 3(a) are the raw ones without any averaging. The corresponding typical and normalized differential signals are respectively shown in Figs. 3(b) and 3(c). We define the SNR as the ratio between the peak-to-peak power variation at the vibrations position and that of the background noise level [7]. Thus, the SNR values of the data presented in Figs. 3(b) and 3(c) are $0.61 \mathrm{~dB}$ and $2.31 \mathrm{~dB}$, respectively. Obviously, our signal processing technique enhance the SNR of the DAS system by $1.70 \mathrm{~dB}$.

Without averaging the Rayleigh traces and based on the sampling theory, the maximum detectable vibration frequency in our experiment is $10 \mathrm{kHz}$ (Nyquist frequency). We proceed to investigate the ability of our setup and signal processing method to detect high vibration frequencies up to the Nyquist one. Figure 4(a) shows the position information of the PZT source when it is vibrating with $5 \mathrm{kHz}$ frequency. Once the vibration location is identified, the vibration frequency can 

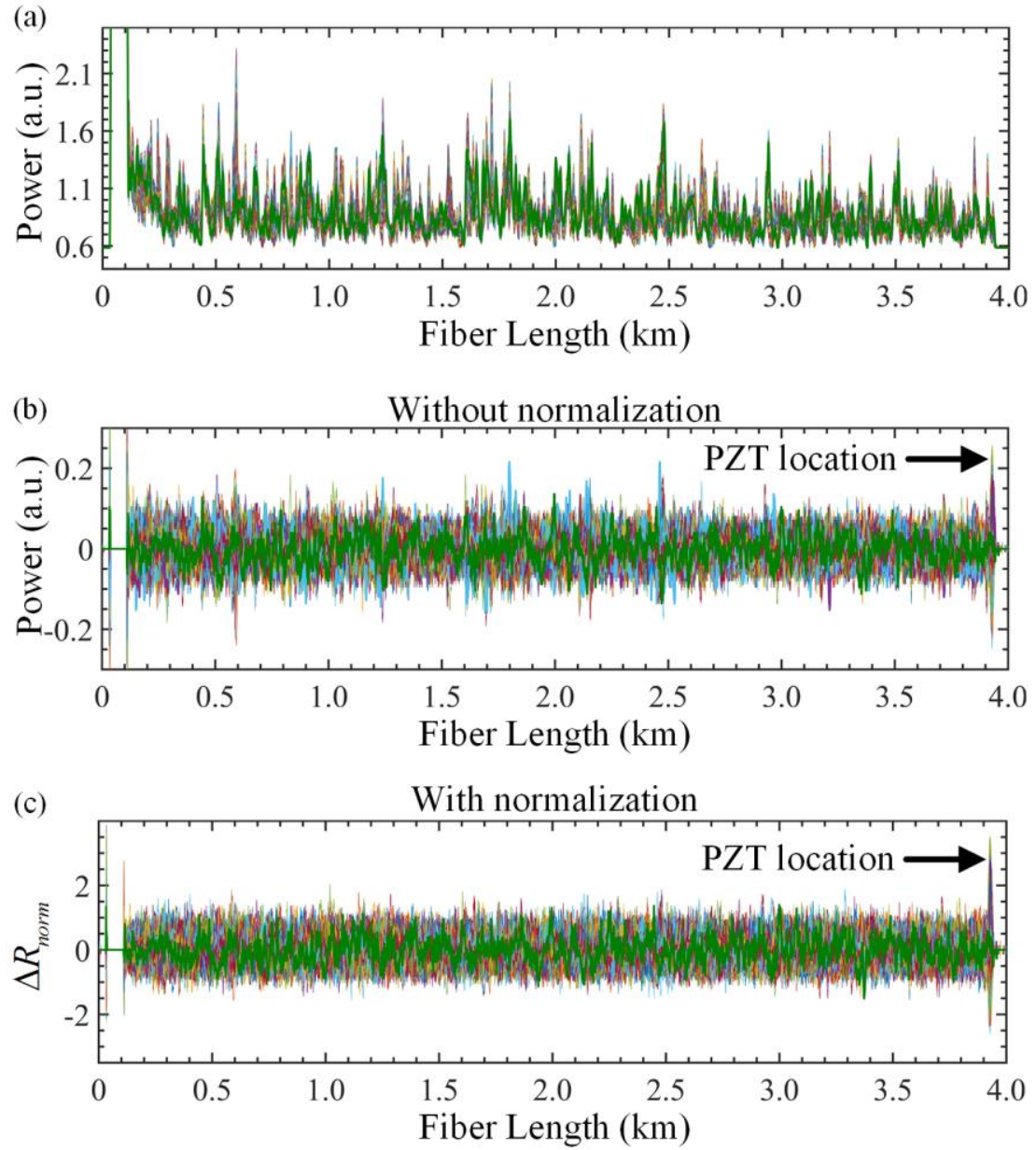

Figure 3. (a) Consecutive Rayleigh traces recorded when the PZT is vibrating. Difference between the Rayleigh traces using the typical differential (b) and normalized differential (c) methods.
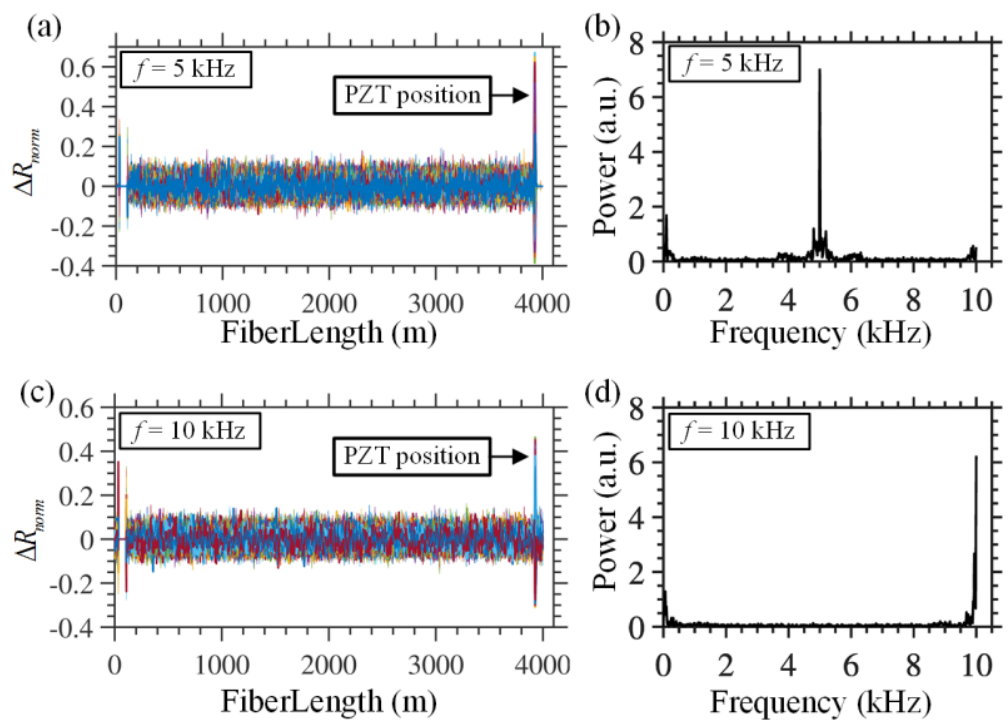

Figure 4. (a) Normalized differential signals of $5 \mathrm{kHz}$ vibration event, and (b) its corresponding power spectrum. (c) Normalized differential signals of $10 \mathrm{kHz}$ vibration event, and (d) its corresponding power spectrum. 
be calculated by applying Fourier transform on the Rayleigh raw traces at this position. Figure 4(b) is the corresponding power spectrum of the $5 \mathrm{kHz}$ vibration event which exhibits accurate frequency detection. A low-frequency noise $(<100$ $\mathrm{Hz}$ ) is also sensed in the power spectrum, which is primarily attributed to inevitable mechanical vibrations in our lab. We further drive the PZT with $10 \mathrm{kHz}$ vibration frequency. As respectively shown in Figs. 4(c) and 4(d), the vibration position and frequency can still efficiently be identified.

\section{CONCLUSION}

In summary, we introduce the normalized differential technique as an efficient tool for enhancing the SNR of the $\Phi$ OTDR-based DAS system. The reported signal processing technique enables noise equalization along the entire optical fiber, which improves the time-domain SNR of the DAS system. We further compare the introduced technique with the typical differential method when handling the data produced by a comparatively noisy DAS system which includes a wide linewidth laser. Our reported method outperforms the conventional one in discovering the vibration position with higher SNR.

\section{ACKNOWLEDGEMENTS}

The authors gratefully acknowledge the financial support provided to this work by the Saudi Aramco under Grant No. RGC/3/3138-01. This work is partially supported by King Abdullah University of Science and Technology (KAUST) baseline funding (BAS/1/1614-01-01), KAUST equipment funding KCR/1/2081-01-01, and GEN/1/6607-01-01.

\section{REFERENCES}

[1] Culshaw, B. and Kersey, A., "Fiber-optic sensing: A historical perspective," J. Light. Technol. 26(9), 1064-1078 (2008).

[2] Bao, X. and Chen, L., "Recent Progress in Distributed Fiber Optic Sensors," Sensors (Switzerland) 12(7), 86018639 (2012).

[3] Juarez, J. C. and Taylor, H. F., "Field test of a distributed fiber-optic intrusion sensor system for long perimeters," Appl. Opt. 46(11), 1968 (2007).

[4] Yamate, T., Fujisawa, G. and Ikegami, T., "Optical Sensors for the Exploration of Oil and Gas," J. Light. Technol. 35(16), 3538-3545 (2017).

[5] Merlo, S., Malcovati, P., Norgia, M., Pesatori, A., Svelto, C., Pniov, A., Zhirnov, A., Nesterov, E. and Karassik, V., "Runways ground monitoring system by phase-sensitive optical-fiber OTDR," 4th IEEE Int. Work. Metrol. AeroSpace, Metroaerosp. 2017 - Proc., 523-529 (2017).

[6] Bao, X., Zhou, D. P., Baker, C. and Chen, L., "Recent Development in the Distributed Fiber Optic Acoustic and Ultrasonic Detection," J. Light. Technol. 35(16), 3256-3267 (2017).

[7] Lu, Y., Zhu, T., Chen, L. and Bao, X., "Distributed vibration sensor based on coherent detection of phase-OTDR," J. Light. Technol. 28(22), 3243-3249 (2010).

[8] Ashry, I., Mao, Y., Alias, M. S., Ng, T. K., Hveding, F., Arsalan, M. and Ooi, B. S., "Normalized differential method for improving the signal-to-noise ratio of a distributed acoustic sensor," Appl. Opt. 58(18), 4933 (2019).

[9] Ren, M., Zhou, D.-P., Chen, L. and Bao, X., "Influence of finite extinction ratio on performance of phase-sensitive optical time-domain reflectometry," Opt. Express 24(12), 13325 (2016). 